\title{
Relativistic Particle Scattering
}

\author{
Stephan De Bièvre` and Gérard G. Emch \\ Departments of Physics and of Mathematics, The University of Rochester, Rochester, NY 14627, \\ USA
}

\begin{abstract}
In the presence of competing relativistic formalisms for interacting particle dynamics, a model-independent axiomatic approach is proposed for the study of the following asymptotic aspects of relativistic classical particle dynamics: the definition of the scattering operator, scattering angle and timedelay, and the specification of a general functional interdependence between the objects so defined.
\end{abstract}

\section{Introduction}

Non-relativistic (i.e. Galilean) scattering theory is usually presented as a comparison, in the asymptotic region, between an interacting-particle dynamics and a free-particle dynamics, with both dynamics cast in the same canonical formalism.

To pursue that approach in the relativistic (i.e. Poincaré) domain however involves some drastic modifications that are required by the Currie-JordanSudarshan no-go theorem [1]; this result asserts, in essence, that the usual Hamiltonian formalism, when coupled with relativistic invariance, allows only free-particle dynamics.

The problem of formulating a relativistic dynamics that allows for interactions has been investigated actively over the last few decades [2], and several models have recently appeared [3]. In contrast with the compelling character of the models studied in the non-relativistic domain, the specific assumptions involved in the relativistic models that have been proposed are often difficult to motivate, interpret or derive unambiguously from general physical principles.

This situation suggests exploring those asymptotic aspects of the dynamics that are dictated by relativistic invariance and should thus hold independently of a detailed description of the intermediary dynamics. We focus in this paper on the scattering of two classical relativistic particles.

Our contribution is twofold: (i) we take full advantage of the fact that actual scattering measurements only involve asymptotically free particles (which the

* Research Assistant NFWO, Belgium 
usual Hamiltonian formalism describes well); (ii) we investigate kinematic quantities (e.g. scattering angle and time-delay) and relations among them, the existence of which does not require at all a Hamiltonian formulation of non-trivial intermediate dynamics that would be prohibited by the no-go theorem.

In Sect. II we isolate a geometric structure that captures the minimal requirements to be imposed on a classical particle-system in Minkowski space; in the framework so provided, we formulate an asymptotic completeness condition that leads directly to our definition of the scattering operator $S$. We also analyse in this section some of the restrictions imposed on $S$ by the requirements of Poincare invariance.

These restrictions are discussed further, in Sect. III, to determine when $S$ can be expressed as a functional of two relativistic invariants; in this context, a relativistic generalization of the notions of scattering angle and time-delay appears naturally.

Finally we make contact, in Sect. IV, with the traditional non-relativistic $S$-operator theory, and with some of the existing relativistic models where a specific intermediary dynamics has been proposed. This shows that the general axiomatic approach presented in this paper has both predictive power and nontrivial realizations.

\section{The Scattering Operator $S$}

In order to emphasize the model-independence of the approach followed here, the notions of particle, of particle system, and of asymptotic completeness are introduced as geometric concepts.

A (relativistic, spinless, classical) particle is identified as a world-line, i.e. as a one-dimensional, time-like, connected, and infinitely extended, submanifold $\gamma^{(k)}$ of the Minkowski space-time $(M, \bar{g})$. A (relativistic) $n$-particle system is then identified as a collection $\Gamma^{n}$ of $n$-tuples $\gamma$ of world-lines such that

$$
g[\gamma] \in \Gamma^{n} \quad \forall g \in G \quad \text { and } \quad \forall \gamma \in \Gamma^{n},
$$

where $\gamma=\left(\gamma^{(1)}, \gamma^{(2)}, \ldots, \gamma^{(n)}\right)$ denotes an arbitrary element of $\Gamma^{n} ; g$ denotes an arbitrary element of the Poincaré group $G$; and $g[\gamma]$ denotes the transform of $\gamma$ under $g$, implemented by the natural action of $G$ on $(M, \bar{g})$.

The introduction of the notion of asymptotic completeness is an adaptation of the intuitive idea one has from the usual non-relativistic theory; in the relativistic case however, it is important to stress the operational role played by the possible observers.

An instantaneous observer $w$ at a point $m \in M$ is identified as a $\bar{g}$-orthogonal basis $\left\{e_{1}, e_{2}, e_{3}, e_{4}\right\}$ of the tangent space [4] $T_{m} M$ to $M$ at $m$, satisfying the following conditions: (i) $\bar{g}\left(e_{i}, e_{i}\right)=+1$ for $i=1,2,3$; (ii) $\bar{g}\left(e_{4}, e_{4}\right)=-c^{2}$ (where $c$ is the speed of light); and (iii) $e_{4}$ is future-pointing.

Given $w$, there exists a unique geodesic coordinate system $\psi^{w}$ on $M$, defined by

$$
\begin{aligned}
& \psi^{w}: m^{\prime} \in M \mapsto(x, t)=\left(x_{1}, x_{2}, x_{3}, t\right) \in \mathbb{R}^{4}, \\
& T_{m} \psi^{w} e_{i}=\partial_{i} \equiv \partial_{x_{i}}, \quad i=1,2,3, \\
& T_{m} \psi^{w} e_{4}=\partial_{4} \equiv \partial_{t}, \\
& \psi^{w}(m)=(0,0,0,0) .
\end{aligned}
$$


In this coordinate system, the line element of the metric $\bar{g}$ is given by

$$
d s^{2}=d x_{1}^{2}+d x_{2}^{2}+d x_{3}^{2}-c^{2} d t^{2} .
$$

For any two instantaneous observers $w$ and $w^{\prime}$, there exists a unique element $g$ of the Poincare group $G$ that transforms $w$ into $w^{\prime}$. This being said, we need not explicitly write, in the sequel, the identification of $M$ with $\mathbb{R}^{4}$ under $\psi^{w}$.

For any choice of $w$, a world-line $\gamma^{(k)}$ can be parametrized by $t: \gamma^{(k)}$ $=\left\{\left(x^{(k)}(t), t\right) \mid t \in \mathbb{R}\right\}$. In particular, the locus of a time-like geodesic $\gamma_{0}^{(k)}$ satisfies

$$
\left.\begin{array}{l}
\gamma_{0}^{(k)} \equiv\left\{\left(x^{(k)}+v^{(k)} t, t\right) \mid t \in \mathbb{R}\right\} \\
\text { for some }\left(x^{(k)}, v^{(k)}\right) \in T \mathbb{R}^{3} \simeq \mathbb{R}^{6} \\
\text { with }\left\|v^{(k)}\right\|^{2}<c^{2} .
\end{array}\right\}
$$

Consequently, the one-particle system $\Gamma_{0}^{1}$ consisting of all time-like geodesic paths can be identified with an open subset of $T \mathbb{R}^{3}$; this identification is, of course, observer-dependent but is nevertheless a relativistically covariant characterization. Alternatively, one can identify in the usual way $\Gamma_{0}^{1}$ with the "phase space" $T^{*} \mathbb{R}^{3}$ by choosing a mass $m$ :

$$
\left.\begin{array}{l}
(x, v) \in T \mathbb{R}^{3} \mapsto(x, p) \in T^{*} \mathbb{R}^{3}, \\
\text { with } p=\left(1-\frac{\|v\|^{2}}{c^{2}}\right)^{-1 / 2} m v .
\end{array}\right\}
$$

The identifications corresponding to different instantaneous observers are related to one another by canonical transformations of $T^{*} \mathbb{R}^{3}$ that define [5] an action of the Poincare group $G$ on $T^{*} \mathbb{R}^{3}$. The ten generators of this action are the functions

$$
\kappa_{j}:(x, p) \in T^{*} \mathbb{R}^{3} \simeq \mathbb{R}^{3} \times \mathbb{R}^{3} \mapsto \kappa_{j}(x, p) \in \mathbb{R}
$$

(with $j=1,2, \ldots, 10$ ) given by:

$$
\left.\begin{array}{lll}
P_{i}(x, p)=p_{i}, & \text { with } & i=1,2,3, \\
H(x, p)=\left[\|p\|^{2} c^{2}+m^{2} c^{4}\right]^{1 / 2}, & & \\
J_{i}(x, p)=(x \times p)_{i}, & \text { with } \quad i=1,2,3, \\
K_{i}(x, p)=H(x, p) x_{i}, & \text { with } \quad i=1,2,3 .
\end{array}\right\}
$$

While the identification of $T^{*} \mathbb{R}^{3}$ with $\Gamma_{0}^{1}$ is observer-dependent, it induces an observer-independent symplectic structure on $\Gamma_{0}^{1}$, than can be canonically recovered, given $m$, from the symplectic structure of $T^{*} M$. We will nevertheless choose to work with the explicit realization of $\Gamma_{0}^{n}$ by $T^{*} \mathbb{R}^{3 n}$. In particular, the twoparticle system $\Gamma_{0}^{2}$ consisting of all couples of time-like geodesic paths is similarly identified with $T^{*} \mathbb{R} \times T^{*} \mathbb{R} \simeq T^{*} \mathbb{R}^{6}$ upon choosing two masses $m^{(1)}$ and $m^{(2)}$. This again leads to a canonical action $\Phi$ of the Poincaré group $G$, acting now on $T^{*} \mathbb{R}^{6}$, with generators

$$
\kappa_{j}\left(x^{(1)}, p^{(1)}, x^{(2)}, p^{(2)}\right)=\sum_{k=1}^{2} \kappa_{j}^{(k)}\left(x^{(k)}, p^{(k)}\right)
$$

with $j=1,2, \ldots, 10$; and $\kappa_{j}^{(k)}$ given by (II.7). 
Let $F\left(T^{*} \mathbb{R}^{6}\right)$ denote the Lie algebra of smooth, real-valued functions on $T^{*} \mathbb{R}^{6}$, with Lie product given by the usual Poisson bracket $\{.,$.$\} (on T^{*} \mathbb{R}^{6}$ ), obtained from the canonical symplectic form $\omega=\sum_{i=1}^{2} \sum_{k=1}^{3} d p_{k}^{(i)} \wedge d x_{k}^{(i)}$ on $T^{*} \mathbb{R}^{6}$, which we shall use throughout (see e.g. III.5). The ten functions $\kappa_{j}$, given in (II.8), span a Lie sub-algebra of $F\left(T^{*} \mathbb{R}^{6}\right)$, isomorphic to the Lie algebra $L(G)$ of the Poincaré group $G$; we denote by

$$
\kappa: \xi \in L(G) \mapsto \kappa_{\xi} \in F\left(T^{*} \mathbb{R}^{6}\right)
$$

the corresponding injection of $L(G)$ into $F\left(T^{*} \mathbb{R}^{6}\right)$, from which the momentum map [4] can be recovered.

$\Gamma_{0}^{2}$ describes a system of two non-interacting particles. In a sense made precise by the no-interaction theorem [1], $\Gamma_{0}^{2}$ is the only two-particle system for which $T^{*} \mathbb{R}^{6}$ can be used in this way as a state space: the dynamics of an interacting twoparticle system $\Gamma^{2}$ cannot be described, in a canonical Hamiltonian manner, on $T^{*} \mathbb{R}^{6}$. Our purpose is to show that $T^{*} \mathbb{R}^{6}$ nevertheless remains useful for the description of scattering.

A two-particle system $\Gamma^{2}$ is said to be asymptotically complete (with respect to an observer $w$ ) if the subset $\bar{D} \cong T^{*} \mathbb{R}^{6}$, defined by the conditions (i) and (ii) below, is open and dense in $T^{*} \mathbb{R}^{6} ; x \equiv\left(x_{-}^{(1)}, p_{-}^{(1)}, x_{-}^{(2)}, p_{-}^{(2)}\right) \in \bar{D}$ if and only if:

(i) There exists exactly one $\gamma=\left(\gamma^{(1)}, \gamma^{(2)}\right) \in \Gamma^{2}$ for which one has, as $t \rightarrow-\infty$,

$$
\left.\begin{array}{l}
\left\|x^{(k)}(t)-x_{-}^{(k)}-\left[E_{-}^{(k)}\right]^{-1} p_{-}^{(k)} c^{2} t\right\| \rightarrow 0, \\
\left\|\frac{d}{d t} x^{(k)}(t)-\left[E_{-}^{(k)}\right]^{-1} p_{-}^{(k)} c^{2}\right\| \rightarrow 0,
\end{array}\right\}
$$

where $k=1,2 ; \gamma^{(k)}=\left\{\left(x^{(k)}(t), t\right) \mid t \in \mathbb{R}\right\} ;$ and

$$
E_{-}^{(k)}=\left\{\left\|p_{-}^{(k)}\right\|^{2} c^{2}+\left[m^{(k)}\right]^{2} c^{4}\right\}^{1 / 2} .
$$

(ii) For every $\gamma$ appearing in (i) there exists exactly one $\left(x_{+}^{(1)}, p_{+}^{(1)}, x_{+}^{(2)}, p_{+}^{(2)}\right) \in T^{*} \mathbb{R}^{6}$ for which one has, as $t \rightarrow+\infty$,

$$
\left.\begin{array}{l}
\left\|x^{(k)}(t)-x_{+}^{(k)}-\left[E_{+}^{(k)}\right]^{-1} p_{+}^{(k)} c^{2} t\right\| \rightarrow 0, \\
\left\|\frac{d}{d t} x^{(k)}(t)-\left[E_{+}^{(k)}\right]^{-1} p_{+}^{(k)} c^{2}\right\| \rightarrow 0,
\end{array}\right\}
$$

where $k=1,2$; and

$$
E_{+}^{(k)}=\left\{\left\|p_{+}^{(k)}\right\|^{2} c^{2}+\left[m^{(k)}\right]^{2} c^{4}\right\}^{1 / 2} .
$$

Note that if $\Gamma^{2}$ is asymptotically complete with respect to one observer $w$, then it is asymptotically complete with respect to all observers; hence our definition of asymptotic completeness is observer-independent and thus refers to an intrinsic property of the system $\Gamma^{2}$. Its meaning is intuitively clear: for almost all couples of geodesic paths $\gamma_{-}=\left(\gamma_{-}^{(1)}, \gamma_{-}^{(2)}\right) \in \Gamma_{0}^{2}$, there exists a unique couple of interacting particles $\gamma=\left(\gamma^{(1)}, \gamma^{(2)}\right) \in \Gamma^{2}$, such that $\gamma^{(k)}$ converges to $\gamma^{(k)}$ in the remote past; moreover there exists a unique couple of geodesic paths $\gamma_{+}=\left(\gamma_{+}^{(1)}, \gamma_{+}^{(2)}\right) \in \Gamma_{0}^{2}$, such that $\gamma^{(k)}$ converges to $\gamma_{+}^{(k)}$ in the distant future. 
Given an asymptotically complete two-particle system $\Gamma^{2}$, the scattering operator $S^{w}$ is defined, for every observer $w$, by

$$
S^{w}\left(x_{-}^{(1)}, p_{-}^{(1)}, x_{-}^{(2)}, p_{-}^{(2)}\right) \equiv\left(x_{+}^{(1)}, p_{+}^{(1)}, x_{+}^{(2)}, p_{+}^{(2)}\right) .
$$

By construction, this map is injective and densely defined in $T^{*} \mathbb{R}^{6}$. The invariance of $\Gamma^{2}$ under the Poincare group $G$ ensures that $\Phi_{g}(\bar{D})=\bar{D}$ for all $g \in G$, where $\Phi$ is the action of $G$, with generators given by (II.8). For the same reason, we also have

$$
S^{w} \circ \Phi_{g}=\Phi_{g} \circ S^{w} \quad \forall g \in G,
$$

and

$$
S^{g[w]}=\Phi_{g} \circ S^{w} \circ \Phi_{g^{-1}} \quad \forall g \in G .
$$

From (II.15) and (II.16), it follows that

$$
S^{g[w]}=S^{w} \equiv S \quad \forall g \in G .
$$

Hence to every asymptotically complete two-particle system $\Gamma^{2}$ is associated an observer-independent, densely defined, operator $S: T^{*} \mathbb{R}^{6} \rightarrow T^{*} \mathbb{R}^{6}$ that commutes with $\Phi$.

Remarks. (i) From the definition of asymptotic completeness, combined with the time-reversal invariance of $\Gamma^{2}$, we have $S(\bar{D})=\bar{D}$, i.e. the space of "out-states" and the space of "in-states" coincide. (ii) On physical grounds, we cannot expect that $\bar{D}=T^{*} \mathbb{R}^{6}$ in general. From non-relativistic two-body scattering, we know indeed that the $S$-operator may not be defined for some "head-on" collisions with specific values of the internal energy of the system $[6,7]$, nor in certain cases where the two particles have zero initial relative velocity; note that, in both of these situations, the internal angular momentum is zero. In the relativistic case, this is expressed by the vanishing of the Pauli-Lubanski four-vector $\left(W, W_{4}\right)$, the definition of which is recalled in (III.2) below. (iii) The length (with respect to the Lorentz metric $\bar{g}$ ) of this four-vector is one of the Casimir invariants of the Poincare group G.

These remarks motivate our assuming, in the sequel, that all scattering information on the systems we want to consider can be obtained from the restriction of $S$ to the open, $G$-stable, dense subset $D$ of $T^{*} \mathbb{R}^{6}$, the elements of which are the points of $T^{*} \mathbb{R}^{6}$ at which the length of the Pauli-Lubanski vector field is different from zero. This is a mathematically convenient assumption although it might, at first sight, seem somewhat overly restrictive from a physical point of view: it excludes all "head-on" collisions, even those for which there is no "trapping." This assumption will nevertheless cause no practical limitation on the theory, since we will assume throughout that the map $S$ is smooth.

\section{The Scattering Angle $\theta$ and the Time-Delay $\tau$}

Our study of the relativistic scattering operator $S$ introduced in the preceding section will take advantage of the structure described in the following lemma concerning the open and dense, $G$-stable domain $D$ in $T^{*} \mathbb{R}^{6}$, on which $S$ is defined. 
Lemma 1. Let $\Phi$ be the action of the Poincare group $G$ on $T^{*} \mathbb{R}^{6}$, with generators $\left\{P_{i}, H, J_{i}, K_{i} \mid i=1,2,3\right\}$ given in (II.8); let $C_{1}$ and $C_{2}$ be the two Casimir invariants of $G$ :

$$
\left.\begin{array}{l}
C_{1} \equiv W_{4}^{2}-\|W\|^{2} \equiv W^{2}, \\
C_{2} \equiv H^{2}-c^{2}\|P\|^{2} \equiv M^{2} c^{4},
\end{array}\right\}
$$

with

$$
\left(W, W_{4}\right) \equiv(-H J+K \times P, c J \cdot P)
$$

and let

$$
D \equiv\left\{a \in T^{*} \mathbb{R}^{6} \mid C_{1}(a) \neq 0\right\} .
$$

Then $\Phi$ defines a foliation of $D$, the leaves of which are orbits of $\Phi$, labeled by the values of the Casimir invariants $C_{1}$ and $C_{2}$.

Proof. The key to the lemma is to show that the orbit $O\left(a_{0}\right)$ of $\Phi \equiv\left\{\Phi_{g} \mid g \in G\right\}$ through an arbitrary point $a_{0} \in D$ is identical to

$$
D\left(a_{0}\right) \equiv\left\{a \in D \mid C_{k}(a)=C_{k}\left(a_{0}\right) ; k=1,2\right\} .
$$

The manifold $D\left(a_{0}\right)$ is 10-dimensional, by virtue of the implicit function theorem and of the functional independence of $C_{1}$ and $C_{2}$ [i.e. the fact that $d C_{1}(a)$ and $d C_{2}(a)$ are linearly independent 1 -forms on $T_{a} D$ for every $\left.a \in D\right]$. Since $C_{1}$ and $C_{2}$ are Casimir invariants, $O\left(a_{0}\right)$ is a submanifold of $D\left(a_{0}\right)$. The tangent space $T_{a} O\left(a_{0}\right)$, to the orbit $O\left(a_{0}\right)$ at $a$, is spanned by the values $X_{j}(a)$ of the 10 vector fields $X_{j}$ determined by $\left.X_{j}\right\lrcorner \omega=d \kappa_{j}$, i.e.

$$
X_{j}(f)(a)=\left\{f, \kappa_{j}\right\}(a)
$$

$\forall f \in F\left(T^{*} \mathbb{R}^{6}\right)$ and $\forall a \in D$; here, as before, $\omega$ is the canonical symplectic form on $T^{*} \mathbb{R}^{6}$. To prove that the orbit $O\left(a_{0}\right)$ is 10 -dimensional, and thus coincides with $D\left(a_{0}\right)$, it is sufficient to prove that the vectors $\left\{X_{j}(a) \mid j=1,2, \ldots, 10\right\}$ are linearly independent. Since the action $\Phi$ is transitive on $O\left(a_{0}\right)$, it is sufficient to prove the independence at any conveniently chosen point of the orbit $O\left(a_{0}\right)$. We know that each orbit intersects the center of mass manifold

$$
D_{\mathrm{CM}} \equiv\left\{a \in D \mid P_{i}(a)=0=K_{i}(a) ; i=1,2,3\right\} \text {. }
$$

Hence, it is sufficient to prove the linear independence of $\left\{X_{j}(a) \mid j=1,2, \ldots, 10\right\}$ for every $a \in D_{\mathrm{CM}}$. To see that this is indeed the case, it is sufficient to note that, with $a \in D_{\mathrm{CM}}$ and $\alpha_{j} \in \mathbb{R}(j=1,2, \ldots, 10)$,

$$
\left.\begin{array}{c}
\left\{\sum_{j} \alpha_{j} \kappa_{j}, p_{i}^{(k)}\right\}(a)=0 \\
\left\{\sum_{j} \alpha_{j} \kappa_{j}, x_{i}^{(k)}\right\}(a)=0
\end{array}\right\} \quad k=1,2 ; i=1,2,3
$$

implies $\alpha_{j}=0 \quad \forall j=1,2, \ldots, 10$. 
The proof of the preceding lemma suggests that the study of the scattering operator $S$ may take advantage of the information already contained in the center of mass manifold $D_{\mathrm{CM}}$. We now prove that $S$ is indeed fully determined by its restriction to $D_{\mathrm{CM}}$.

Lemma 2. Let $D$ and $D_{\mathrm{CM}}$ be defined as in Lemma 1. If two diffeomorphisms $S_{1}$ and $S_{2}$ of $D$ satisfy

$$
\begin{gathered}
S_{i} \circ \Phi_{g}=\Phi_{g} \circ S_{i} \quad \forall g \in G \quad(i=1,2), \\
S_{1}(a)=S_{2}(a) \quad \forall a \in D_{\mathrm{CM}},
\end{gathered}
$$

then $S_{1}=S_{2}$ on $D$.

Proof. We already noticed, in the proof of Lemma 1, that every orbit of $\Phi$ intersects $D_{\mathrm{CM}}$. Hence, for each $a \in D$, there exists some $g \in G$ such that $a_{0} \equiv \Phi_{g^{-1}}(a) \in D_{\mathrm{CM}}$. We have then, upon using (III.8) and (III.9):

$$
S_{1}(a)=S_{1}\left(\Phi_{g}\left(a_{0}\right)\right)=\Phi_{g} \circ S_{1}\left(a_{0}\right)=\Phi_{g} \circ S_{2}\left(a_{0}\right)=S_{2}\left(\Phi_{g}\left(a_{0}\right)\right)=S_{2}(a) .
$$

In the remainder of this section, we study the conditions under which the relativistic scattering operator $S$ is uniquely determined by two real-valued functions on $D$ that are constant on each orbit of $\Phi$. By Lemma 1 , these functions thus depend only on the two Casimir invariants $C_{1}$ and $C_{2}$; one of them will be seen to be the CM scattering angle $\theta$, while the other will be interpreted as the $\mathrm{CM}$ time-delay $\tau$.

Theorem 1. Let $D$ and $D_{\mathrm{CM}}$ be as in Lemma 1 ; and let $f_{A}$ and $f_{B}$ be the $\mathbb{R}^{3}$-valued functions defined on $T^{*} \mathbb{R}^{6}$ by

$$
\begin{gathered}
f_{A}\left(x^{(1)}, p^{(1)}, x^{(2)}, p^{(2)}\right) \equiv E^{(1)} x^{(1)}-E^{(2)} x^{(2)}, \\
f_{B}\left(x^{(1)}, p^{(1)}, x^{(2)}, p^{(2)}\right) \equiv E^{(2)} p^{(1)}-E^{(1)} p^{(2)},
\end{gathered}
$$

with

$$
E^{(k)}\left(x^{(k)}, p^{(k)}\right) \equiv\left\{\left\|p^{(k)}\right\|^{2} c^{2}+\left[m^{(k)}\right]^{2} c^{4}\right\}^{1 / 2}
$$

Let finally $S$ be a diffeomorphism of $D$ satisfying

$$
\begin{aligned}
& \Phi_{g} \circ S=S \circ \Phi_{g} \quad \forall g \in G, \\
& \kappa_{\xi} \circ S=\kappa_{\xi} \quad \forall \xi \in L(G) .
\end{aligned}
$$

There exists then a unique pair of functions

$$
\theta: D \rightarrow[0,2 \pi) \text { and } \tau: D \rightarrow \mathbb{R}
$$

satisfying

$$
\theta \circ \Phi_{g}=\theta \quad \text { and } \quad \tau \circ \Phi_{g}=\tau \quad \forall g \in G,
$$

and such that, on the center of mass manifold $D_{\mathrm{CM}}$,

$$
\begin{aligned}
& f_{A} \circ S=\cos \theta f_{A}+\sin \theta f_{A} \times \frac{W}{\|W\|}+\frac{2 \tau c^{2}}{M c^{2}} f_{B} \circ S, \\
& f_{B} \circ S=\cos \theta f_{B}+\sin \theta f_{B} \times \frac{W}{\|W\|} .
\end{aligned}
$$


Proof. Let $a=\left(x^{(1)}, p^{(1)}, x^{(2)}, p^{(2)}\right) \in D_{\mathrm{CM}}$. We have then

$$
\left.\begin{array}{l}
p^{(2)}=-p^{(1)}, H(a)=E^{(1)}+E^{(2)} \\
J(a)=\left[1+\frac{E^{(1)}}{E^{(2)}}\right] x^{(1)} \times p^{(1)}
\end{array}\right\}
$$

with $E^{(k)}$ as in (III.12). Let further $\hat{a} \equiv S(a)$, i.e.

$$
\left(\hat{x}^{(1)}, \hat{p}^{(1)}, \hat{x}^{(2)}, \hat{p}^{(2)}\right)=S\left(x^{(1)}, p^{(1)}, x^{(2)}, p^{(2)}\right) .
$$

From (III.14), we have $\kappa_{j}(\hat{a})=\kappa_{j}(a)$ (with $j=1,2, \ldots, 10$ ), and thus: $\hat{a} \in D_{\mathrm{CM}},\left\|\hat{p}^{(1)}\right\|$ $=\left\|p^{(1)}\right\|, \hat{x}^{(1)} \times \hat{p}^{(1)}=x^{(1)} \times p^{(1)} \neq 0$. Consequently, there exists $\theta(a) \in[0,2 \pi)$ such that

$$
\hat{p}^{(1)}=\cos [\theta(a)] p^{(1)}+\sin [\theta(a)] p^{(1)} \times \frac{J(a)}{\|J(a)\|} .
$$

The desired transformation property (III.18) follows then straightforwardly from the definition (III.11, 12) of $f_{B}$, the condition (III.14), the fact that $a$ and $\hat{a}$ belong to $D_{\mathrm{CM}}$, and the relation (III.21) just established.

Note further that any vector $A \in \mathbb{R}^{3}$ such that $A \times \hat{p}^{(1)}=x^{(1)} \times p^{(1)}$ can always be written as

$$
A=\cos [\theta(a)] x^{(1)}+\sin [\theta(a)] x^{(1)} \times \frac{J(a)}{\|J(a)\|}+\alpha(a) \hat{p}^{(1)} .
$$

Equation (III.20), and the immediate consequences we drew from it, thus imply that $\hat{x}^{(1)}$ can be written in the form given by (III.22). We use again the fact that $a$ and $\hat{a}$ belong to $D_{\mathrm{CM}}$, and the definition (III.10,12) of $f_{A}$, to show that the desired transformation property (III.17) follows directly from (III.22) with $\tau$ determined by $\frac{2 E^{(1)} \alpha(a)}{H(a)}=2 \tau c^{2} / M c^{2}$.

We have thus established the transformation properties (III.17) and (III.18), and the existence of $\theta$ and $\tau$ on $D_{\mathrm{CM}}$. We still have to prove that $\theta$ and $\tau$ can be defined everywhere on $D$, and satisfy (III.16).

To complete the proof of the theorem, notice that $a \in D_{\mathrm{CM}}$ and $g \in G$ with $\Phi_{g}(a) \in D_{\mathrm{CM}}$ imply that $g \in \mathrm{SO}(3)$, the rotation group generated by $\left\{J_{i} \mid i=1,2,3\right\}$. From the relations (III.21) and (III.22 with $A=\hat{x}^{(1)}$ ), together with the invariance (III.13) of $S$ under $\Phi$, one obtains

$$
\begin{gathered}
\theta \circ \Phi_{g}(a)=\theta(a) \text { and } \tau \circ \Phi_{g}(a)=\tau(a), \\
\forall g \in \operatorname{SO}(3) \text { and all } a \in D_{\mathrm{CM}}
\end{gathered}
$$

(compare with III.16). Together with the fact that every orbit of $\Phi$ intersects $D_{\mathrm{CM}}$, (III.23) ensures the existence and uniqueness of an extension of $\theta$ and $\tau$ to $D$, subject to the condition (III.16).

The functions $\theta$ and $\tau$, appearing in Theorem 1, play a central role in the theory. Mathematically, as a consequence of(III.16) and of Lemma 1, they are functions of the Casimir invariants only. Physically, $\theta$ is readily interpreted as the center of 
mass scattering angle, and we now indicate how $\tau$ is to be viewed as the center of mass time-delay.

When two particles move uniformly along straight lines, they have a time of closest approach. In a scattering process, we denote the time by $t_{-}$(respectively $t_{+}$) for the in-state (respectively for the out-state). The time-delay is then operationally defined as the quantity $\left(t_{+}-t_{-}\right)$. In a relativistic framework, the time-delay is an observer-dependent quantity. We compute it now in the center of mass frame. Let $\left(x^{(1)}, p^{(1)}, x^{(2)}, p^{(2)}\right)$ be the in-state; $t_{-}^{\mathrm{CM}}$ is then determined by the equation

$$
\frac{d}{d t}\left\|x^{(1)}(t)-x^{(2)}(t)\right\|^{2}=0 \quad \text { at } \quad t=t_{-}^{\mathrm{CM}}
$$

where

$$
x^{(k)}(t)=x^{(k)}+\frac{c^{2} t}{E^{(k)}} p^{(k)} \quad k=1,2
$$

Hence

$$
\left[x^{(1)}\left(t_{-}^{\mathrm{CM}}\right)-x^{(2)}\left(t_{-}^{\mathrm{CM}}\right)\right] \cdot\left[\frac{c^{2} p^{(1)}}{E^{(1)}}-\frac{c^{2} p^{(2)}}{E^{(2)}}\right]=0 .
$$

Upon using the definition (III.6) of the center of mass manifold, i.e.

$$
p^{(1)}+p^{(2)}=0 \quad \text { and } \quad E^{(1)} x^{(1)}+E^{(2)} x^{(2)}=0,
$$

we obtain, from (III.26):

$$
x^{(k)}\left(t_{-}^{\mathrm{CM}}\right) \cdot p^{(k)}=0 \quad k=1,2 ;
$$

and thus, upon substituting in (III.25):

$$
t_{-}^{\mathrm{CM}}=\frac{x^{(k)} \cdot p^{(k)}}{\left\|p^{(k)}\right\|} \frac{E^{(k)}}{c^{2}\left\|p^{(k)}\right\|}, \quad k=1,2 .
$$

The value of $t_{+}^{\mathrm{CM}}$ is similarly computed from the out-state $S\left(x^{(1)}, p^{(1)}, x^{(2)}, p^{(2)}\right)$. frame:

From the definition (III.10-12) of $f_{A}$ and $f_{B}$ we have, in the center of mass

$$
\frac{f_{A} \cdot f_{B}}{\left\|f_{B}\right\|^{2}}=\frac{2 c^{2}}{H} \frac{x^{(k)} \cdot p^{(k)}}{\left\|p^{(k)}\right\|} \frac{E^{(k)}}{c^{2}\left\|p^{(k)}\right\|}, \quad k=1,2 .
$$

Upon comparing (III.29) and (III.30), we have:

$$
t_{-}^{\mathrm{CM}}=\frac{H}{2 c^{2}} \frac{f_{A} \cdot f_{B}}{\left\|f_{B}\right\|^{2}} .
$$

Similarly

$$
t_{+}^{\mathrm{CM}}=\frac{H}{2 c^{2}} \frac{\left(f_{A} \circ S\right) \cdot\left(f_{B} \circ S\right)}{\left\|f_{B} \circ S\right\|^{2}} .
$$

Finally, from (III.17-18):

$$
\frac{\left(f_{A} \circ S\right) \cdot\left(f_{B} \circ S\right)}{\left\|f_{B} \circ S\right\|^{2}}=\frac{f_{A} \cdot f_{B}}{\left\|f_{B}\right\|^{2}}+\frac{2 \tau c^{2}}{M c^{2}} .
$$


Since $H=M c^{2}$ on $D_{\mathrm{CM}}$, (III.31-33) give:

$$
\tau=t_{+}^{\mathrm{CM}}-t_{-}^{\mathrm{CM}}
$$

i.e. $\tau$ is indeed to be interpreted as the center of mass time-delay.

In Theorem 1 we assumed that the scattering operator $S$ not only commutes with the action $\Phi$ of the Poincare group $G$ on $T^{*} \mathbb{R}^{6}$ (see III.13), but that it moreover preserves the generators of this action (see III.14). When $S$ is assumed to be a canonical transformation, the supplementary assumption (III.14) however is an immediate consequence of a general conservation law, akin to the Jacobi theorem [4]. We have indeed the following result.

Lemma 3. If $S: T^{*} \mathbb{R}^{6} \rightarrow T^{*} \mathbb{R}^{6}$ is a symplectic diffeomorphism of $D$, and if $S \circ \Phi_{g}$ $=\Phi_{g} \circ S$ for all $g \in G$, then $\left(\kappa_{\xi} \circ S\right)(a)=\kappa_{\xi}(a)$ for all $\xi \in L(G)$ and all $a \in D$.

Proof. Choose $\xi \in L(G)$, and let $\{\exp (\lambda \xi) \mid \lambda \in \mathbb{R}\} \subset G$. Since $S$ commutes with the action $\Phi$ of $G$, we have, for all $f \in F\left(T^{*} \mathbb{R}^{6}\right)$ and all $a \in D$ :

$$
\left.\frac{d}{d \lambda} f\left(\Phi_{\exp (-\lambda \xi)} \circ S \circ \Phi_{\exp (\lambda \xi)}(a)\right)\right|_{\lambda=0}=0,
$$

i.e.

$$
\left\{f \circ S, \kappa_{\xi}\right\}(a)-\left\{f, \kappa_{\xi}\right\}(S a)=0 .
$$

Since $S$ is symplectic, this implies

$$
\left\{f, \kappa_{\xi} \circ S^{-1}-\kappa_{\xi}\right\}(S a)=0 .
$$

Since (III.37) holds for all $f \in T^{*} \mathbb{R}^{6}$ and all $a \in D$, and since, moreover, $S(D)=D$ and $D$ connected, there exists $c_{\xi} \in \mathbb{R}$ such that

$$
\left(\kappa_{\xi} \circ S\right)(a)=\kappa_{\xi}(a)+c_{\xi} \quad \forall a \in D .
$$

Notice now that for each $\kappa_{j}$ in (II.8) there exist $i$ and $l$ such that

$$
\left\{\kappa_{i}, \kappa_{l}\right\}=\kappa_{j} .
$$

We therefore have, from (III.38-39):

$$
\left.\begin{array}{rl}
\kappa_{j} & =\left\{\kappa_{i}, \kappa_{l}\right\}=\left\{\kappa_{i}+c_{i}, \kappa_{l}+c_{l}\right\} \\
& =\left\{\kappa_{i} \circ S, \kappa_{l} \circ S\right\}=\kappa_{j} \circ S=\kappa_{j}+c_{j},
\end{array}\right\}
$$

i.e. $c_{j}=0 \forall j=1,2, \ldots, 10$, and thus $c_{\xi}=0 \forall \xi \in L(G)$.

It follows from Theorem 1 and Lemma 3 that every symplectic scattering operator satisfies (III.17-18). In the remainder of this section, we determine the necessary and sufficient conditions on $\theta$ and $\tau$ for $S$ to be symplectic. We will see, in particular, that the scattering angle $\theta$ and the time-delay $\tau$ are expressed in terms of a single function $\varphi$ of the Casimir invariants $C_{1}$ and $C_{2}$ (defined in III.1-2).

We need to introduce some notation. For $f \in F\left(T^{*} \mathbb{R}^{6}\right)$, we denote by $X_{f}$ the Hamiltonian vector field defined by $\left.X_{f}\right\lrcorner \omega=d f$, where $\omega$ is the canonical symplectic form on $T^{*} \mathbb{R}^{6}$ :

$$
X_{f}=\left(\frac{\partial f}{\partial p^{(1)}},-\frac{\partial f}{\partial x^{(1)}}, \frac{\partial f}{\partial p^{(2)}},-\frac{\partial f}{\partial x^{(2)}}\right) \text {. }
$$


The flow of a (globally integrable) vector field $X$ is written $\left\{F_{t}^{X} \mid t \in \mathbb{R}\right\}$, and we use the notations $\exp \bar{X}$ (respectively $\exp \bar{f}$ ) for $F_{1}^{X}$ (respectively $\exp \bar{X}_{f}$ ). We now investigate the form of the scattering operator $S$ in cases where it can be written as $S=\exp \bar{\phi}$ with $\phi$ a function on $T^{*} \mathbb{R}^{6}$ satisfying

$$
\phi(a)=\widetilde{\phi}\left(C_{1}(a), C_{2}(a)\right) \quad \forall a \in D,
$$

where

$$
\tilde{\phi}:(x, y) \in \mathbb{R}^{2} \mapsto \tilde{\phi}(x, y) \in \mathbb{R}
$$

is a smooth function on $\mathbb{R}^{2}$, and $C_{1}, C_{2}$ are the two Casimir invariants defined in (III.1-2). In these cases, $S$ is symplectic and commutes with the action $\Phi$.

In the forthcoming computations, we use the following simplifying notations (valid $\forall a \in D$ ):

$$
\begin{aligned}
& c_{1}(a) \equiv C_{2}^{-1}(a) C_{1}(a) \quad \text { and } \quad c_{2}(a) \equiv C_{2}(a) \\
& \varphi\left(c_{1}(a), c_{2}(a)\right) \equiv \tilde{\phi}\left(C_{1}(a), C_{2}(a)\right) \\
& \varphi_{1}(a) \equiv \frac{\partial \varphi}{\partial c_{1}}\left(c_{1}(a), c_{2}(a)\right) \\
& \varphi_{2}(a) \equiv \frac{\partial \varphi}{\partial c_{2}}\left(c_{2}(a), c_{2}(a)\right)
\end{aligned}
$$

Note also the following identities

$$
\left.\begin{array}{l}
X_{\varphi}=\varphi_{1} X_{c_{1}}+\varphi_{2} X_{c_{2}}, \\
\left\{c_{1}, c_{2}\right\}=0,\left\{\varphi_{1}, \varphi_{2}\right\}=0, \\
{\left[\varphi_{1} X_{c_{1}}, \varphi_{2} X_{c_{2}}\right]=0 .}
\end{array}\right\}
$$

Hence, if $S$ is of the form

$$
\left.\begin{array}{l}
S=\exp \bar{\phi} \text { with } \\
\phi \in F\left(T^{*} \mathbb{R}^{6}\right) \text { such that (III.42) holds, }
\end{array}\right\}
$$

then

$$
S=\exp \overline{\varphi_{1} X_{c_{1}}} \circ \exp \overline{\varphi_{2} X_{c_{2}}} .
$$

Lemma 4. The scattering operator $S$ is of the form (III.46) if and only if the following two conditions are met. (i) $S$ satisfies (III.17-18); and (ii) the scattering angle $\theta$ and the time-delay $\tau$ satisfy the relations

$$
\begin{gathered}
\theta=2 \varphi_{1} \frac{\|W\|}{M c^{2}}=\frac{\partial \varphi}{\partial\left(\|W\| / M c^{2}\right)}, \\
\tau=2 \varphi_{2} M c^{2}=\frac{\partial \varphi}{\partial\left(M c^{2}\right)},
\end{gathered}
$$

where $\varphi, \varphi_{1}$, and $\varphi_{2}$ are defined as in (III.44). 
Proof. One first verifies, by a straightforward computation, that, for any smooth function $f: T^{*} \mathbb{R}^{6} \rightarrow \mathbb{R}^{3}$ satisfying

$$
\left\{f_{i}, J_{j}\right\}=\varepsilon_{i j k} f_{k} \text { with } i, j, k=1,2,3,
$$

one has, for all $a \in D_{\mathrm{CM}}$ :

$$
X_{c_{1}}{ }^{n}(f)(a)=\left(\delta_{J}^{n} f\right)(a),
$$

where $\delta_{J}$ is defined for all $\psi: T^{*} \mathbb{R}^{6} \rightarrow \mathbb{R}^{3}$ by

$$
\left(\delta_{J} \psi\right)(a)=-2 \psi(a) \times J(a) .
$$

In particular, if the smooth function $f: T^{*} \mathbb{R}^{6} \rightarrow \mathbb{R}^{3}$ satisfies

$$
f(a) \cdot J(a)=0 \quad \forall a \in D_{\mathrm{CM}},
$$

(III.51) reduces to:

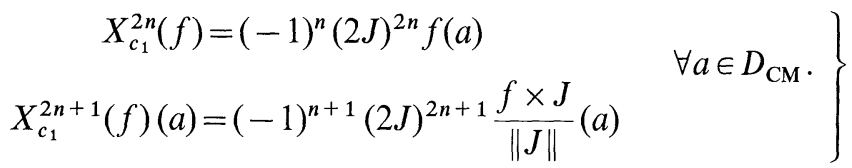

Notice, in particular, that $f_{A}$ and $f_{B}$ in (III.10-11) satisfy the condition (III.53).

With $S$ satisfying (III.46), we have upon using (III.47):

$$
\left.\begin{array}{l}
f_{A} \circ S=\left(f_{A} \circ \exp \overline{\varphi_{2} X_{c_{2}}}\right) \circ \exp \overline{\varphi_{1} X_{c_{1}}} \\
f_{B} \circ S=\left(f_{B} \circ \exp \overline{\varphi_{2} X_{c_{2}}}\right) \circ \exp \overline{\varphi_{1} X_{c_{1}}} .
\end{array}\right\}
$$

We now restrict all the forthcoming equations to $D_{\mathrm{CM}}$; upon using the explicit form (III.10-11) of $f_{A}$ and $f_{B}$, one computes

$$
\left.\begin{array}{l}
f_{A} \circ \exp \overline{\varphi_{2} X_{c_{2}}}=f_{A}+4 \varphi_{2} c^{2} f_{B}, \\
f_{B} \circ \exp \overline{\varphi_{2} X_{c_{2}}}=f_{B} .
\end{array}\right\}
$$

Upon inserting this in (III.55) and using (III.54) one finds:

$$
\begin{aligned}
f_{A} \circ S= & \cos \left(\frac{2 \varphi_{1}\|W\|}{M c^{2}}\right) f_{A}+\sin \left(\frac{2 \varphi_{1}\|W\|}{M c^{2}}\right) f_{A} \times \frac{W}{\|W\|}, \\
& +4 \varphi_{2} c^{2} f_{B} \circ S \\
f_{B} \circ S= & \cos \left(\frac{2 \varphi_{1}\|W\|}{M c^{2}}\right) f_{B}+\sin \left(\frac{2 \varphi_{1}\|W\|}{M c^{2}}\right) f_{B} \times \frac{W}{\|W\|} .
\end{aligned}
$$

The "if" part of the lemma then follows from the comparison of (III.17-18) and (III.57-58). The converse implication is obvious from the above discussion, together with Lemma 2.

Theorem 2. Let $S: D \rightarrow D\left(\subset T^{*} \mathbb{R}^{6}\right)$ be a diffeomorphism such that $\Phi_{g} \circ S=S \circ \Phi_{g}$ $\forall g \in G$, then $S$ is symplectic if and only if it is of the form (III.46), i.e. $S=\exp \bar{\phi}$, where the smooth, real-valued function $\phi$ depends on $a \in D$ only through the two Casimir invariants $C_{1}$ and $C_{2}$ of the Poincaré group $G$. 
Proof. If $S$ satisfies (III.46) it is symplectic. To prove the converse note that if $S$ is symplectic, Theorem 1 and Lemma 3 establish that $S$ satisfies (III.17-18). From Lemma 4 we know that it is sufficient for our purpose to prove that (III.48-49) holds. Furthermore, since $S$ is symplectic, the following 1 -form $\beta$ is closed:

$$
\beta=S^{*} \alpha-\alpha,
$$

where $\alpha$ is the canonical 1 -form

$$
\alpha=\sum_{k=1}^{2} p^{(k)} \cdot d x^{(k)},
$$

and $S^{*} \alpha$ is defined by

$$
S^{*} \alpha=\sum_{k=1}^{2}\left(p^{(k)} \circ S\right) \cdot\left(d x^{(k)} \circ S\right) .
$$

From the definition of $D_{\mathrm{CM}} C D \subset T^{*} \mathbb{R}^{6}$, one verifies that $D_{\mathrm{CM}}$ inherits from $\left(T^{*} \mathbb{R}^{6}, \omega\right)$ the structure of a symplectic manifold; since $S$ is symplectic on $D$ and maps $D_{\mathrm{CM}}$ into itself, its restriction to this symplectic manifold is also symplectic. Upon using (III.17-18), one then evaluates the corresponding restriction of $\beta$ to $T D_{\mathrm{CM}}$; one finds that, for every $v_{a} \in T_{a} D_{\mathrm{CM}}$ :

$$
\beta\left(v_{a}\right)=d \eta\left(v_{a}\right)-\left\{\theta(a)\left(d c_{1}^{1 / 2}\right)\left(v_{a}\right)+\tau(a)\left(d c_{2}^{1 / 2}\right)\left(v_{a}\right)\right\}
$$

with

$$
\eta=c_{1}^{1 / 2} \theta+c_{2}^{1 / 2} \tau-\sum_{k=1}^{2} \frac{\left[m^{(k)} c^{2}\right]^{2}}{E^{(k)}} \tau .
$$

Since $\beta$ is closed, (III.48-49) now follow immediately.

\section{Discussion}

Having obtained, in the preceding sections, the general structure of the classical relativistic scattering operator $S$, we want now to touch briefly upon the following three problems: (i) the non-relativistic limit; (ii) the connection with quantum scattering cross-sections computed, in the usual manner, to lowest order of perturbation; (iii) the actual construction of non-trivial realizations of the scattering theory delineated in the main body of the paper.

The invariance group of non-relativistic two-particle systems is the Galilei group $G_{0}$. A reasoning similar to that followed in Sect. II leads to a densely defined scattering operator $S: T^{*} \mathbb{R}^{6} \rightarrow T^{*} \mathbb{R}^{6}$ that commutes with the natural action $\Phi$ of $G_{0}$ on $T^{*} \mathbb{R}^{6}$. The generators of that action are

$$
\begin{aligned}
& P=p^{(1)}+p^{(2)} \\
& H=\frac{\left\|p^{(1)}\right\|^{2}}{2 m^{(1)}}+\frac{\left\|p^{(2)}\right\|^{2}}{2 m^{(2)}}=\frac{\|P\|^{2}}{2 M}+\frac{\|P\|^{2}}{2 \mu}, \\
& J=X \times P+x \times p=L+l \\
& K=M X
\end{aligned}
$$


where

$$
\left.\begin{array}{rl}
M & =m^{(1)}+m^{(2)} ; M \mu=m^{(1)} m^{(2)}, \\
M X & =m^{(1)} x^{(1)}+m^{(2)} x^{(2)} ; x=x^{(1)}-x^{(2)}, \\
M p & =m^{(2)} p^{(1)}-m^{(1)} p^{(2)}
\end{array}\right\}
$$

The two Casimir invariants are now $\|l\|^{2}$ and $\|p\|^{2}$. The analogues of Lemmas III.1 and III.2 hold with:

$$
D_{0} \equiv\left\{a \in T^{*} \mathbb{R}^{6} \mid l \neq 0\right\}
$$

If the non-relativistic scattering operator $S_{0}$ preserves the values of all generators of $G_{0}$, one proves as in Theorem III.1 that

$$
\begin{gathered}
x \circ S_{0}=\cos \theta_{0} x+\sin \theta_{0} \frac{l \times x}{\|l\|}+\frac{\tau_{0}}{\mu} p \circ S_{0}, \\
p \circ S_{0}=\cos \theta_{0} p+\sin \theta_{0} \frac{l \times p}{\|l\|} .
\end{gathered}
$$

These equations now hold everywhere on $D_{0}$; the scattering angle $\theta_{0}$ and the timedelay $\tau_{0}$ are (again) functions of the Casimir invariants alone. When $S_{0}$ is symplectic, one proves along the lines followed in the proof of Lemma III.3, that nine of the 10 generators (IV.2) - namely $P_{i}, J_{i}$, and $K_{i}$ with $i=1,2,3$ - are conserved in the scattering process; for the tenth - namely $H$-one can only prove, however, that $H \circ S=H+c_{H}$, where $c_{H}$ is an arbitrary constant in $\mathbb{R}$. Nevertheless, in the non-relativistic framework, an intermediate Hamiltonian dynamics on $T^{*} \mathbb{R}^{6}$ is available, and mild conditions are known $[6,7]$ under which the following facts can be proven: $S_{0}$ exists; $c_{H}=0$; and the symplectic operator $S_{0}$ can be written as $S_{0}=\exp \bar{\psi}_{0}$, where the form of $\psi_{0} \equiv \psi_{0}\left(\|l\|^{2},\|p\|^{2}\right)$ is evidently to be determined from the interaction potential. Not only can one obtain, as a consequence of the above, that (IV.4-5) hold, but one can moreover show that the non-relativistic scattering angle $\theta_{0}$ and time-delay $\tau_{0}$ satisfy

$$
\begin{gathered}
\theta_{0}=2 \psi_{1}\|l\|=\frac{\partial \psi_{0}}{\partial\|l\|}, \\
\tau_{0}=2 \psi_{2} \mu=\frac{\partial \psi_{0}}{\partial\left(\|p\|^{2} / 2 \mu\right)} .
\end{gathered}
$$

Since we know now the general structure of both the Galilei- and the Poincareinvariant scattering operators $S_{0}$ and $S$, we are in a position to discuss the sense in which the relativistic operator $S$ is actually approximated, on some part of its domain, by the non-relativistic operator $S_{0}$. The use of the latter is justified only when both particles move slowly in the center of mass frame, i.e. when for both $k=1$ and $k=2$ :

$$
\left\|p^{(k)}\right\| c \ll m^{(k)} c^{2}
$$


When these two conditions are satisfied, we obtain from (III.16) and Lemma 1 (with $\left.M=m^{(1)}+m^{(2)}\right)$ :

$$
\begin{aligned}
& \theta \cong \theta\left(\left\|l_{\mathrm{CM}}\right\|^{2}, M c^{2}+\frac{1}{2 \mu}\left\|p_{\mathrm{CM}}\right\|^{2}\right), \\
& \tau \simeq \tau\left(\left\|l_{\mathrm{CM}}\right\|^{2}, M c^{2}+\frac{1}{2 \mu}\left\|p_{\mathrm{CM}}\right\|^{2}\right),
\end{aligned}
$$

so that $\theta$ and $\tau$ are now essentially functions of the Casimir invariants of the Galilei group $G_{0}$. In that sense $S$ behaves indeed as a non-relativistic scattering operator.

We now return to the relativistic theory. From $\theta=\theta\left(C_{1}, C_{2}\right)$, the differential scattering cross-section $\sigma$ is defined in complete analogy with the non-relativistic theory [8]:

$$
\sigma\left(\theta, M^{2} c^{4}\right)=\frac{1}{2} \cdot \frac{1}{\sin \theta}\left|\frac{d s^{2}}{d \theta}\right|,
$$

where the impact parameter in the center of mass is given by

$$
s^{2}=\frac{1}{\left\|p^{(1)}\right\|^{2}} \cdot \frac{W^{2}}{M^{2} c^{4}} .
$$

Elastic cross-sections obtained in perturbative quantum field theory are of the form (IV.11), i.e. at fixed $\theta$, they depend on $M c^{2}$ only. The specific form of this function will, of course, depend on the choice of the dynamics; the determination of this functional dependence is an open challenge on which we intend to focus in a separate publication, using the "constraints" approach followed elsewhere [2,9] for bound-state problems. The following particular - but explicit - realization of the general scheme proposed in Sects. II and III is a step in that direction; this model is given here because it proves that our scheme actually covers non-trivial scattering situations.

To be specific, we now investigate the existence and structure of the scattering operator in a case where $\Gamma^{2}$ is constructed using a Hamiltonian constraint dynamics. We concentrate on the simplest model available $[9,10]$, a brief description of which we first recall and then sharpen.

Let $T^{*}(M \times M)$ be the cotangent bundle of $M \times M$, where $M$ is the fourdimensional Minkowski space; an arbitrary point in the 16-dimensional manifold $T^{*}(M \times M)$ is denoted $a=\left(x^{(1)}, p^{(1)}, x^{(2)}, p^{(2)}\right)$. A 13-dimensional submanifold $\Xi \subset T^{*}(M \times M)$ is defined as follows. Let $m^{(1)}$ and $m^{(2)}$ be two positive constants, to be interpreted as the masses of the particles; $V$ be a real-valued function on $\mathbb{R}$; and

$$
\left.\begin{array}{rl}
P & \equiv p^{(1)}+p^{(2)} ; p \equiv p^{(1)}-p^{(2)}, \\
X & \equiv \frac{1}{2}\left[x^{(1)}+x^{(2)}\right] ; x \equiv \frac{1}{2}\left[x^{(1)}-x^{(2)}\right], \\
\mu & \equiv \frac{m^{(1)} m^{(2)}}{m^{(1)}+m^{(2)}} .
\end{array}\right\}
$$


Note that $P, p, X$, and $x$ are now four-vectors; we write $P^{2}$ (respectively $P \cdot x$ ) for $\bar{g}^{\mu v} P_{\mu} P_{v}$ (respectively $\bar{g}^{\mu \nu} P_{\mu} x_{v}$ ). With

$$
\left.\begin{array}{l}
\Xi_{1} \equiv P^{2}+p^{2}+2\left\{\left[m^{(1)}\right]^{2}+\left[m^{(2)}\right]^{2}\right\}+8 \mu V\left(x^{2}-\frac{(P \cdot x)^{2}}{P^{2}}\right), \\
\Xi_{2} \equiv P \cdot p+\left\{\left[m^{(1)}\right]^{2}-\left[m^{(2)}\right]^{2}\right\} \\
\Xi_{3} \equiv P \cdot x,
\end{array}\right\}
$$

the submanifold $\Xi$ is defined by

$$
\Xi \equiv\left\{a \in T^{*}(M \times M) \mid \Xi_{i}(a)=0 ; i=1,2,3\right\} .
$$

Note that $\Xi$ is stable under the natural action of the Poincare group $G$ on $T^{*}(M \times M)$.

Let $\omega$ denote the canonical symplectic form on $T^{*}(M \times M) ; X_{i}(i=1,2,3)$ be the vector fields on $T^{*}(M \times M)$ defined by

$$
\left.X_{i}\right\lrcorner \omega=d \Xi_{i}, \quad i=1,2,3,
$$

and $Y$ be a vector field on $\Xi$ satisfying

$$
\begin{aligned}
& Y \in \operatorname{Span}\left(X_{1}, X_{2}, X_{3}\right), \\
& Y\left(\Xi_{i}\right)=0 \quad i=1,2,3 .
\end{aligned}
$$

Up to a constant $u \in \mathbb{R}$, these conditions determine $Y$ uniquely, namely

$$
Y=u\left[\frac{1}{4} X_{1}-\frac{1}{2} \frac{P \cdot p}{P^{2}} X_{2}\right]
$$

moreover, the flow of $Y$ on $\Xi$ is Poincaré-invariant. Let now $\pi^{(k)}(k=1,2)$ be the natural projections

$$
\pi^{(k)}:\left(x^{(1)}, p^{(1)}, x^{(2)}, p^{(2)}\right) \in T^{*}(M \times M) \rightarrow x^{(k)} \in M .
$$

Whenever $p^{(1)}$ and $p^{(2)}$ are time-like, two world lines $\gamma^{(k)}(k=1,2)$ in $M$ are obtained as the projections, through $\pi^{(k)}(k=1,2)$, of the flow lines of $Y$ in $\Xi$. The collection $\Gamma_{V}^{2}$ of all couples of world lines obtained in this way is stable under the natural action of the Poincare group on $M: \Gamma_{V}^{2}$ is a two-particle system in the sense of Sect. II.

We next will show that, under certain conditions on $V$, this system is asymptotically complete.

We first note, from (IV.18), that $\dot{P}=0$, where the dot (') refers to the differentiation with respect to the flow parameter along the flow lines of $Y$.

Given $\left(x^{(1)}, x^{(2)}, p^{(1)}, p^{(2)}\right) \in \Xi$, consider an instantaneous observer $w$ such that, in the corresponding coordinate system, $P=\left(0,0,0, P_{4}\right)$, i.e. $\|P\|^{2}=0$, where we keep the notation $\|A\|$ for the Euclidean norm of the spatial part, with respect to $w$, of a four-vector $A$.

In this coordinate system, the differential equations of the motion are particularly simple; the relative motion is described by

$$
\ddot{x}_{j}=-u^{2} \mu \frac{\partial V}{\partial x_{j}}\left(\|x\|^{2}\right), \quad j=1,2,3 .
$$


It is a particular feature of the constraints (IV.14), chosen to define this simple model, that Eqs. (IV.20) are of the same form as the equations describing the motion of a non-relativistic particle in a central field of force: this is precisely what makes the model easily tractable. Indeed the asymptotic behaviour of $x$, in the relativistic model considered here, can be obtained from known results in nonrelativistic scattering theory. Specifically, for non-relativistic central force motions, suitable conditions on $V$ are known [6] under which the existence of the scattering operator can be established; we thus choose for the function $V$, appearing in the constraints (IV.14), a function that satisfies these known conditions; in particular, we assume henceforth that $V(r)$ decreases faster than $r^{-1}$ at infinity.

These results ensure the existence of a complete set of asymptotically free solutions of Eqs. (IV.20) describing the relative motion $x$ in the present model.

Moreover, in the coordinate system where $\|P\|^{2}=0$, the vector field $Y$ on $\Xi$ yields, in addition to (IV.20), the differential equations of motion

$$
\left(1+\frac{P \cdot p}{P^{2}}\right) \dot{x}_{j}^{(1)}+\left(1-\frac{P \cdot p}{P^{2}}\right) \dot{x}_{j}^{(2)}=0, \quad j=1,2,3 .
$$

Together with the constraint $\Xi_{2}=0$, and the fact that $\dot{P}=0$, (IV.21) implies that

$$
\left(1+\frac{P \cdot p}{P^{2}}\right) x_{j}^{(1)}+\left(1-\frac{P \cdot p}{P^{2}}\right) x_{j}^{(2)} \equiv \varrho_{j}, \quad j=1,2,3
$$

are conserved in the course of the motion.

Since, furthermore, the $x_{j}$ are asymptotically free, (IV.22) implies that the $x_{j}^{(k)}$ are asymptotically free $(j=1,2,3 ; k=1,2)$.

While the above argument was carried in the coordinate system where $\|P\|^{2}=0$, the $G$-invariance of $\Gamma_{V}^{2}$ allows us to conclude that $\Gamma_{V}^{2}$ is asymptotically complete, in the sense defined in Sect. II. This establishes the existence of the scattering operator on an open and dense, $G$-stable domain $D$ in $T^{*} \mathbb{R}^{6}$.

We now show that the conditions imposed on $S$ in Theorem 1 are realized in the particular model under consideration. We only have to show that (III.14) holds, i.e. that the generators of the action $\Phi$ of $G$ are conserved in the scattering process.

We first notice that

$$
\left.\begin{array}{c}
\varepsilon \equiv \frac{\|\dot{x}\|^{2}}{2 u^{2} \mu}+V\left(\|x\|^{2}\right), \\
\lambda_{j} \equiv(x \times p)_{j} / 2 \quad j=1,2,3
\end{array}\right\}
$$

are first integrals of (IV.20), with $\dot{x}_{j}=\frac{1}{2} u p_{j}(j=1,2,3)$. Upon taking into account the choice of our coordinate system, in which $\|P\|^{2}=0$, we can rewrite (IV.23) in the form:

$$
\left.\begin{array}{c}
\varepsilon=\frac{1}{2 \mu}\left\|p^{(1)}\right\|^{2}+V\left(\|x\|^{2}\right), \\
\lambda_{j}=\left(x \times p^{(1)}\right)_{j}, \quad j=1,2,3 .
\end{array}\right\}
$$


Moreover (IV.22) can be rewritten as

$$
p_{4}^{(1)} x_{j}^{(1)}+p_{4}^{(2)} x_{j}^{(2)}=\frac{1}{2} P_{4} \varrho_{j}, \quad j=1,2,3 .
$$

Furthermore, recall that we are working on $\Xi$, where the constraints $\Xi_{1}=0=\Xi_{2}$ imply, for $k=1,2$ :

$$
p_{4}^{(k)}=\left\{\left[E^{(k)}\right]^{2}+2 \mu V\left(\|x\|^{2}\right)\right\}^{1 / 2}
$$

with

$$
E^{(k)}=\left\{\left\|p^{(k)}\right\|^{2}+\left[m^{(k)}\right]^{2}\right\}^{1 / 2} .
$$

Upon inserting (IV.26-27) into (IV.25), we obtain the asymptotic relations

$$
E^{(1)} x_{j}^{(1)}+E^{(2)} x_{j}^{(2)} \simeq \frac{1}{2} P_{4} \varrho_{j}, \quad j=1,2,3,
$$

valid as $\|x\| \rightarrow \infty$. The desired conclusion, namely that (III.14) holds, is then obtained as the immediate consequence of $\dot{P}=0$, (IV.23-24) and (IV.28).

Under these circumstances, we can write down the expressions for the scattering angle $\theta$ and the time-delay $\tau$. We have first

$$
\theta\left(\|\lambda\|^{2}, \varepsilon\right)=\pi-2 \int_{r_{0}}^{\infty} d r r^{-2}\left[\frac{2 \mu(\varepsilon-V)}{\|\lambda\|^{2}}-\frac{1}{r^{2}}\right]^{-1 / 2},
$$

where $r_{0}$ is the largest positive root of the expression under the square root.

A similar expression can be written down for $\tau$, giving $\tau$ as a function of $\|\lambda\|^{2}$ and $\varepsilon$.

It is moreover known [7] that there exists a function $\psi$ of $\|\lambda\|$ and $\varepsilon$ such that

$$
\begin{aligned}
& \theta\left(\|\lambda\|^{2}, \varepsilon\right)=\frac{\partial \psi}{\partial\|\lambda\|}, \\
& \tau\left(\|\lambda\|^{2}, \varepsilon\right)=\frac{\partial \psi}{\partial \varepsilon} .
\end{aligned}
$$

Compare to (III.48-49); and notice that $\|\lambda\|$ and $\varepsilon$ are functions of the Casimir operators $C_{1}=W^{2}$ and $C_{2}=M^{2} c^{4}$.

The value for $\tau$ appearing in (IV.31) is the flow-parameter-delay when we choose $u=2 \mu^{-1}$, such that $\dot{x}_{j}=P_{j} / m$. In order to obtain the center-of-mass timedelay, one has to choose $\frac{1}{2} u=\frac{E^{(1)}+E^{(2)}}{E^{(1)} E^{(2)}}$, i.e. make the substitution

$$
\tau \rightarrow \mu^{-1} \frac{E^{(1)} E^{(2)}}{E^{(1)}+E^{(2)}} \tau \text {. }
$$

Remarking also that $\|\lambda\|=\frac{1}{2} W / M c^{2}$ [since $\left.x=\frac{1}{2}\left(x_{1}-x_{2}\right)\right]$, one verifies that (IV.30-31) can be rewritten, with the substitution (IV.32):

$$
\left.\begin{array}{l}
\theta=\frac{\partial(2 \psi)}{\partial\left(W / M c^{2}\right)}, \\
\tau=\frac{\partial(2 \psi)}{\partial\left(M c^{2}\right)} .
\end{array}\right\}
$$

Comparing this to (III.48-49), we conclude that $S$ is symplectic. 
Finally, upon taking the non-relativistic limit of this model, we obtain a nonrelativistic two-particle system with interparticle potential $V$. The non-relativistic $S$ operator is again determined by (IV.30-31). As we already pointed out, in the preceding section, all the dynamic information contained in $S$ is given by (IV.30-31). Hence the non-relativistic and the relativistic scattering operators for this model differ only through the kinematics; in particular, the non-relativistic and the relativistic differential scattering cross-sections calculated from (IV.24-25) will have, for this model, the same angular dependence. It is to be expected that the general scheme proposed in the main body of this paper for relativistic particle scattering will transcend such limitations.

The simple model, discussed in the third part of this section, nevertheless shows that - in spite of the no-go theorem, ruling out the existence of an interacting relativistic dynamics in $T^{*} \mathbb{R}^{6}$ - actual scattering situations are covered by the general scheme proposed in Sects. II and III for the description, within $T^{*} \mathbb{R}^{6} \simeq \Gamma_{0}^{2}$, of relativistic particle scattering.

Acknowledgements. The authors would like to express their appreciation to Professor L. Streit for his hospitality at the Zentrum für interdisziplinäre Forschung der Universität Bielefeld where part of this work was done. They also want to acknowledge here the stimulating comments they received from Professors B. French, C. Günther, and R. Lavine.

\section{References}

1. Currie, D.G., Jordan, T.F., Sudarshan, E.C.G.: Relativistic invariance and Hamiltonian theories of interacting particles. Rev. Mod. Phys. 35, 350-375 (1963)

2. A survey and further bibliography can be found in: Kerner, E.H.: The theory of action-at-adistance in relativistic particle dynamics, a reprint collection. New York: Gordon and Breach 1972; and in Relativistic action-at-a-distance: Classical and quantum aspects. Lecture Notes in Physics, Vol. 162, Llosa, J., (ed.), Berlin, Heidelberg, New York: Springer 1982

3. Balachandran, A.P., et al.: Unified geometrical approach to relativistic particle dynamics. J. Math. Phys. 25, 167-176 (1984) and references therein

4. We follow the notation of Abraham, R., Marsden, J.E.: Foundations of Mechanics. London: The Benjamin Cummings Publishing 1978

5. Sudarshan, E.C.G., Mukunda, N.: Classical dynamics: A modern perspective. London: John Wiley 1974

6. Simon, B.: Wave operators for classical particle scattering. Commun. Math. Phys. 23, 37-48 (1971)

7. Narnhofer, H., Thirring, W.: Classical scattering transformation in classical mechanics. Phys. Rev. A 23, 1683-1697 (1981)

8. Goldstein, H.: Classical mechanics. Reading, MA: Addison-Wesley 1980

9. Todorov, I.T.: Communication of the JINR, E2-10125. Dubna (1976); Komar, A.: Interacting relativistic particles. Phys. Rev. D 18, 1887-1892 (1978); Kihlberg, A., Marnelius, R., Mukunda, N.: Relativistic potential models as systems with constraints and their interpretation. Phys. Rev. D 23, 2201-2209 (1981)

10. Molotkov, V.V., Todorov, I.T.: Gauge dependence of worldlines and invariance of the $S$-matrix in relativistic particle mechanics. Commun. Math. Phys. 79, 111-122 (1981)

Communicated by H. Araki

Received January 29, 1985; in revised form May 14, 1985 
